Innovative Applications of O.R.

\title{
Stable solutions for optimal reinsurance problems involving risk measures
}

\author{
Alejandro Balbás*, Beatriz Balbás, Antonio Heras
}

University Carlos III of Madrid, CL. Madrid, 126, 28903 Getafe, Madrid, Spain

University of Castilla la Mancha, Avda. Real Fábrica de Seda, s/n, 45600 Talavera, Toledo, Spain

University Complutense of Madrid, Somosaguas-Campus, 28223 Pozuelo de Alarcón Madrid, Spain

\section{A R T I C L E I N F O}

\section{Article history:}

Received 3 May 2010

Accepted 19 May 2011

Available online 27 May 201

\section{Keywords:}

Optimal reinsurance

Risk measure

Sensitivity

optimal retentio

Stop-loss reinsurance
A B S T R A C T

The optimal reinsurance problem is a classic topic in actuarial mathematics. Recent approaches conside a coherent or expectation bounded risk measure and minimize the global risk of the ceding company under adequate constraints. However, there is no consensus about the risk measure that the insurer must use, since every risk measure presents advantages and shortcomings when compared with others.

This paper deals with a discrete probability space and analyzes the stability of the optimal reinsurance with respect to the risk measure that the insurer uses. We will demonstrate that there is a "stable optimal retention" that will show no sensitivity, insofar as it will solve the optimal reinsurance problem for many risk measures, thus providing a very robust reinsurance plan. This stable optimal retention is a stop los contract, and it is easy to compute in practice. A fast linear time algorithm will be given and a numerical example presented.

\section{Introduction}

Since the paper by Artzner et al. (1999) introduced the coherent measures of risk many authors have further extended the discus sion, which shows the importance that this topic is achieving in finance and insurance. Among others, Goovaerts et al. (2004) intro duced the consistent risk measures, also studied in Burgert and Rüschendorf (2006), Frittelli and Scandolo (2005) analyzed risk measures for stochastic processes, and Rockafellar et al. (2006) de fined the deviations and the expectation bounded risk measures.

Classical actuarial and financial problems have been then revis ited using risk measures beyond the variance. Among others, Nakano (2004) and Balbás et al. (2010) drew on risk measures when pricing in incomplete markets, Mansini et al. (2007) and Schied (2007) dealt with portfolio choice problems, and Annaert et al. (2009) checked the efficiency of the classical portfolio insurance problem if the risk level is given by the value at risk $(\mathrm{VaR})$ or the conditional value at risk $(\mathrm{CVaR})$.

The optimal reinsurance problem is a main issue in actuarial science. A common approach attempts to minimize some measure of the first insurer risk after reinsurance. Seminal papers by Borch (1960) and Arrow (1963) used the variance as a risk measure and proved that the stop loss reinsurance minimizes the retained risk if

* Corresponding author at: University Carlos III of Madrid, CL. Madrid, 126, 28903 Getafe, Madrid, Spain.

E-mail addresses: alejandro.balbas@uc3m.es (A. Balbás), beatriz.balbas@uclm.es (B. Balbás), aheras@ccee.ucm.es (A. Heras). premiums are calculated following the expected value premium principle.

The subsequent research followed similar ideas and tried to take into account more general risk measures and premium princi ples, which may give optimal contracts other than stop loss Recently, Gajec and Zagrodny (2004) considered more general symmetric and even asymmetric risk functions such as the abso lute deviation and the truncated variance of the retained loss, un der the standard deviation premium principle. Kaluszka (2005) studied reinsurance contracts with many convex premium princi ples (exponential, semi deviation and semi variance, Dutch, dis tortion, etc.). Other well known financial risk measures such as the $V a R$ or the tail value at risk (TVaR) are also being considered. For example, Kaluszka (2005) uses the TVaR as a premium principle and Cai and Tan (2007) calculate the optimal retention for a stop loss reinsurance by considering the $V a R$ and the conditional tail expectation risk measures (CTE), under the expected value pre mium principle.

The most recent papers have finally incorporated coherent and or expectation bounded risk measures in the objective function to be minimized by the ceding company. Along with the paper of $\mathrm{Ca}$ and Tan (2007) above, other interesting examples are Cai et al. (2008), Balbás et al. (2009) or Bernard and Tian (2009). The differ ences among their approaches are caused by the insurer behavior Very complete information may be found in the survey of Centeno and Simoes (2009).

Despite the interest of the problem, as far as we know there are no analyses focusing on the stability of the optimal reinsurance. This should be an important topic since the optimality of many 
reinsurance plans will critically depend on the risk measure and the pricing principle. There is no consensus about the risk measure that the insurer must use, since every risk measure presents advantages and shortcomings when compared with others.

This paper considers that the reinsurer's premium principle is given by a convex function and deals with the optimal reinsurance problem if risk is measured by coherent and expectation bounded risk measures. ${ }^{1}$ The focus is on the stability in the large of the opti mal retention plan with respect to the chosen risk measure. "Stabil ity in the large" is used in the sense of Samuelson (1947), i.e., we will analyze whether the optimal contract remains constant as the risk measure becomes more and more risk adverse (the risk measure increases).

The paper's outline is as follows. Section 2 will present the basic conditions and properties of the risk measure $\rho$ to be used. Section 3 provides our general optimal reinsurance problem. We will pres ent the problem in a discrete probability space. Actually, this sim plifies the mathematical exposition, and every probability space admits a discrete approximation which achieves as much accuracy as needed. Many actuarial and financial analyses deal with discrete probability spaces (see Benati, 2003; Konno et al., 2005; Mansini et al., 2007, or Miller and Ruszczynski, 2008, among many others), since this is not a restriction in practice. The proposed optimal reinsurance problem seems to be quite flexible and general, since it allows us to incorporate many particular situations such as bud get constraints, the maximization of the insurer expected wealth, etc. The most important results in Section 3 are Theorem 2 and Corollary 4 , since they characterize the optimal retention by means of Karush Kuhn Tucker (KKT) like conditions and permit us to introduce the "stable optimal retention", 2 which will solve the problem for all of the risk measures with a subgradient satisfying adequate properties. Therefore, the stable optimal retention may be understood as a robust optimal reinsurance plan.

Section 4 is devoted to computing in practice the stable optimal retention. Here we will assume that the reinsurer uses a linear value principle, containing the expected value premium principle as a particular case. Of course it is not necessary, since practical optimal ity conditions have been given in a much more general framework, but the specific solution of the optimization problem depends on the premium principle we take, and considering more than one would significantly enlarge the paper. As already indicated, previ ous literature measuring the insurer risk by a general risk measure is still limited, so it seems to be natural and of interest to analyze concrete problems by taking the most used premium principle.

The most important result of this section is Theorem 8 , because it gives explicit expressions for the stable optimal retention and the KKT multipliers of the problem. According to Theorem 8, the stable optimal retention is a stop loss reinsurance.

Theorem 8 is used in Section 5 so as to introduce a fast algorithm that gives the stable optimal retention in numerical applications. The algorithm is not time consuming since there is a linear relation ship between its computational complexity and the complexity of the portfolio of insurance policies. An illustrative numerical exam ple is also provided, which clarifies how to use the algorithm in practice and shows the robustness of the given reinsurance, in the sense that most of the usual risk measures lead to this solution.

The last section of the paper summarizes the most important conclusions.

\footnotetext{
${ }^{1}$ Insurance premiums are usually given by convex functions, see, for instance, Deprez and Gerber (1985).

2 Actually, the given KKT - like conditions are not exactly the same as the standard KKT conditions of the problem, and that is the reason why we say "KKT - like". Nevertheless, they are necessary and sufficient optimality conditions, and are generated by the KKT conditions of an equivalent optimization problem presented in Balbás et al. (2009). Further details may be found in that paper.
}

\section{Preliminaries and notations}

As usual, consider the probability space $(\Omega, \mathscr{F}, \mathbb{P})$ composed of the set of "states of the world" $\Omega$, the $\sigma$ algebra $\mathscr{F}$ and the proba bility measure $\mathbb{P}$. As said above, we will be dealing with a discrete framework, so $\Omega$ will be composed of a finite number of elements,

$\Omega \quad\left\{\omega_{1}, \omega_{2}, \ldots, \omega_{n}\right\}$.

We will consider the probability of every single event

$p_{i} \quad \mathbb{P}\left(\omega_{i}\right)>0$,

$i=1,2, \ldots, n$.

Denote by $\mathbb{E}(y)$ the mathematical expectation of every random variable $y$, and denote by $L^{2}$ the Hilbert space of $\mathbb{R}$ valued random variables $y$ on $\Omega$ endowed with the norm

$\|y\|_{2} \quad\left(\mathbb{E}\left(|y|^{2}\right)\right)^{1 / 2}$

for every $y \in L^{2}$. $^{3}$

Let $[0, T]$ be a time interval. From an intuitive point of view, one can interpret that every $y \in L^{2}$ may represent the wealth at $T$ of an arbitrary insurer. Let

$\rho: L^{2} \rightarrow \mathbb{R}$

be the general risk function that an insurer uses in order to control the risk level of his final wealth at $T$. Denote by

$\Delta_{\rho} \quad\left\{z \in L^{2} ; \quad \mathbb{E}(y z) \leqslant \rho(y), \forall y \in L^{2}\right\}$.

We will assume that $\Delta_{\rho}$ is convex and compact, and

$\rho(y) \operatorname{Max}\left\{\mathbb{E}(y z): z \in \Delta_{\rho}\right\}$,

holds for every $y \in L^{2}$. Furthermore, we will also suppose that the constant random variable $z=1$ is in $\Delta_{\rho}$ and

$\Delta_{\rho} \subset\left\{z \in L^{2} ; \mathbb{E}(z) \quad 1\right\}$.

Summarizing, we have:

Assumption 1. The set $\Delta_{\rho}$ given by (2) is convex and compact, (3) holds for every $y \in L^{2}, z=1$ is in $\Delta_{\rho}$, and (4) holds.

The assumption above is closely related to the representation theorem of risk measures stated in Rockafellar et al. (2006). Fol lowing their ideas, it is easy to prove that the fulfillment of Assumption 1 holds if and only if $\rho$ satisfies:

(a) $\rho(y+k) \quad \rho(y) \quad k$

for every $y \in L^{2}$ and $k \in \mathbb{R}$.

(b) $\rho(\alpha y) \quad \alpha \rho(y)$

for every $y \in L^{2}$ and $\alpha>0$.

(c) $\rho\left(y_{1}+y_{2}\right) \leqslant \rho\left(y_{1}\right)+\rho\left(y_{2}\right)$

for every $y_{1}, y_{2} \in L^{2}$.

(d) $\rho(y) \geqslant \mathbb{E}(y)$

for every $y \in L^{2}$.

\footnotetext{
${ }^{3}$ Actually, $\Omega$ being discrete and containing $n \in \mathbb{N}$ elements the dimension of $L^{2}$ is finite and equals $n$. Thus, $L^{2}=L^{p}$ for every $p \in[1, \infty]$ and the norm $\|\cdot\|_{2}$ above is equivalent to the norm $\|\cdot\|_{p}$. Though we have chosen $p=2$, every $p \in[1, \infty]$ may play the same role.

4 Actually, the properties above are almost similar to those used by Rockafellar et al. (2006) in order to introduce their expectation bounded risk measures.
} 
It is easy to see that if $\rho$ satisfies properties (a) (d) then it is also coherent in the sense of Artzner et al. (1999) if and only if

$$
\Delta_{\rho} \subset L_{+}^{2} \quad\left\{z \in L^{2} ; \mathbb{P}(z \geqslant 0) \quad 1\right\} .
$$

Particular interesting examples are the conditional value at risk (CVaR) of Rockafellar et al. (2006), the weighted conditional value at risk (WCVaR) of Cherny (2006), the dual power transform (DPT) of Wang (2000) and the Wang measure (Wang, 2000), among many others. Furthermore, following the original idea of Rockafellar et al. (2006) to identify their expectation bounded risk measures and their deviation measures, it is easy to see that

$$
\rho(y) \quad \sigma(y) \quad \mathbb{E}(y),
$$

satisfies (a) (d) if $\sigma: L^{2} \rightarrow \mathbb{R}$ is a deviation, that is, if $\sigma$ satisfies (b), (c),

(e) $\sigma(y+k) \quad \sigma(y)$

for every $y \in L^{2}$ and $k \in \mathbb{R}$, and

(f) $\sigma(y) \geqslant 0$

for every $y \in L^{2}$.

Among many others, a particular example is the classical $p$ deviation for every $p \in[1, \infty)$, given by

$\sigma_{p}(y) \quad\left[\mathbb{E}\left(|\mathbb{E}(y) \quad y|^{p}\right)\right]^{1 / p}$,

or the downside $p$ semi deviation, given by

$\sigma_{p}(y) \quad\left[\mathbb{E}\left(|\operatorname{Max}\{\mathbb{E}(y) \quad y, 0\}|^{p}\right)\right]^{1 / p}$.

The classical separation theorems allow us to prove that there is a one to one mapping $\rho \leftrightarrow \Delta_{\rho}$ between the risk measures satisfying Assumption 1 that are coherent and the set of convex and compact subsets of $L^{2}$ such that $z=1$ is in $\Delta_{\rho}$, and (4) and (9) hold. Further more (3) shows that this mapping is increasing, i.e., $\rho_{1}(y) \leqslant \rho_{2}(y)$ holds for every $y \in L^{2}$ if and only if $\Delta_{\rho_{1}} \subset \Delta_{\rho_{2}}$ holds. Accordingly, the maximum coherent risk measure satisfying Assumption 1 is that $\Gamma$ associated with the set

$\Delta_{\Gamma} \quad\left\{z \in L_{+}^{2}: \mathbb{E}(z) \quad 1\right\}$.

It is easy to see that the risk measure $\Gamma$ is

$\Gamma(y) \quad \operatorname{Min}\left\{y\left(\omega_{i}\right): i \quad 1,2, \ldots, n\right\}$

for every $y \in L^{2}$. Similarly, $y \rightarrow \mathbb{E}(y)$ is the minimum risk measure satisfying the conditions above, since $\Delta_{\mathbb{E}} \quad\{1\}$. Thus

$\Gamma(y) \geqslant \rho(y) \geqslant \mathbb{E}(y)$,

holds for every $y \in L^{2}$ and every coherent $\rho$ satisfying Assumption 1 .

Finally, once again the separation theorems allow us to prove that every convex combination

$\rho \quad \sum_{i=1}^{m} w_{i} \rho_{i}$

of risk measures satisfying (5) (9) also satisfies (5) (9), and

$\Delta_{\rho} \quad \sum_{i=1}^{m} w_{i} \Delta_{\rho_{i}}$

holds.

\section{Optimal reinsurance: General problem and optimality conditions}

Consider that the insurance company receives the fixed amount $S_{0}$ (premium) and will have to pay the random variable $y_{0} \in L_{+}^{2}$ within a given period $[0, T]$ (claims). Without loss of generality we will assume that $\mathbb{P}\left(y_{0}>0\right) \quad 1$, since the absence of claims is an unrealistic situation in practice.

Suppose that a reinsurance contract is signed in such a way that the company will only pay $y \in L^{2}$, whereas the reinsurer will pay $y_{0} y$. If the reinsurer premium principle is given by the convex and increasing function,

$\pi: L^{2} \rightarrow \mathbb{R}$

such that $\pi(0)=0$, and $S_{1}>0$ is the largest amount that the insurer would like to pay for the contract, then the insurance company will choose $y$ (optimal retention) so as to solve the bi criteria optimiza tion problem

$\left\{\begin{array}{l}\operatorname{Min} \rho_{0}\left(S_{0} \quad y \quad \pi\left(y_{0} \quad y\right)\right), \\ \operatorname{Max} \mathbb{E}\left(S_{0} \quad y \quad \pi\left(y_{0} \quad y\right)\right), \\ \pi\left(y_{0} \quad y\right) \leqslant S_{1}, \\ 0 \leqslant y \leqslant y_{0},\end{array}\right.$

$\rho_{0}$ being a coherent risk measure that satisfies Assumption 1. Con ditions $\pi(0)=0$ and $S_{1}>0$ imply that $y=y_{0}$ satisfies the constraint, so (15) is always feasible (Theorem 2 below will show that it is also bounded and solvable). Notice that, if desired, constraint $\pi\left(y_{0} \quad y\right) \leqslant S_{1}$ may be removed without modifying (15), since $\pi$ is increasing and therefore it is sufficient to choose $S_{1}>\pi\left(y_{0}\right)$.

First of all let as see that the multiobjective optimization prob lem (15) is convex.

Lemma 1. With the notations of (15) we have that the three functions

$L^{2} \ni y \rightarrow \rho_{0}\left(S_{0} \quad y \quad \pi\left(y_{0} \quad y\right)\right) \in \mathbb{R}$,

$L^{2} \ni y \rightarrow \mathbb{E}\left(S_{0} \quad y \quad \pi\left(y_{0} \quad y\right)\right) \in \mathbb{R}$

and

$L^{2} \ni y \rightarrow \pi\left(y_{0} \quad y\right) \in \mathbb{R}$

are convex.

Proof. Let us prove that (16) is convex since the remaining cases are analogous. Thus, suppose that $y_{1}, y_{2} \in L^{2}$ and $0 \leqslant \lambda \leqslant 1$.

$$
\begin{aligned}
& \rho_{0}\left(S_{0} \quad\left(\lambda y_{1}+\left(\begin{array}{ll}
1 & \lambda
\end{array}\right) y_{2}\right) \quad \pi\left(y_{0} \quad\left(\lambda y_{1}+\left(\begin{array}{ll}
1 & \lambda
\end{array}\right) y_{2}\right)\right)\right) \\
& \rho_{0}\left(\lambda\left(\begin{array}{ll}
S_{0} & y_{1}
\end{array}\right)+\left(\begin{array}{ll}
1 & \lambda
\end{array}\right)\left(S_{0} \quad y_{2}\right) \quad \pi\left(\lambda\left(\begin{array}{ll}
y_{0} & y_{1}
\end{array}\right)+\left(\begin{array}{lll}
1 & \lambda
\end{array}\right)\left(\begin{array}{ll}
y_{0} & y_{2}
\end{array}\right)\right)\right) .
\end{aligned}
$$

Since $\pi$ is convex we have that

$$
\left.\pi\left(\begin{array}{ll}
y_{0} & y_{1}
\end{array}\right)+\left(\begin{array}{lll}
1 & \lambda
\end{array}\right)\left(\begin{array}{ll}
y_{0} & y_{2}
\end{array}\right)\right) \geqslant \lambda \pi\left(\begin{array}{lll}
y_{0} & y_{1}
\end{array}\right) \quad\left(\begin{array}{ll}
1 & \lambda
\end{array}\right) \pi\left(y_{0} \quad y_{2}\right) .
$$

$\rho_{0}$ is decreasing because it is coherent. ${ }^{5}$ Hence

$$
\begin{aligned}
& \left.\rho_{0}\left(S_{0} \quad\left(\begin{array}{llllll}
\lambda y_{1} S_{0} & y & \pi\left(y_{0}\right. & y
\end{array}\right) y_{2}\right) \quad \pi\left(y_{0} \quad\left(\lambda y_{1}+\left(\begin{array}{ll}
1 & \lambda
\end{array}\right) y_{2}\right)\right)\right) \\
& \leqslant \rho_{0}\left(\lambda\left(\begin{array}{ll}
S_{0} & y_{1}
\end{array}\right)+\left(\begin{array}{lll}
1 & \lambda
\end{array}\right)\left(S_{0} \quad y_{2}\right) \quad \lambda \pi\left(\begin{array}{lll}
y_{0} & y_{1}
\end{array}\right) \quad\left(\begin{array}{ll}
1 & \lambda
\end{array}\right) \pi\left(y_{0} \quad y_{2}\right)\right) .
\end{aligned}
$$

Finally, since $\rho_{0}$ is convex,

$$
\begin{aligned}
& \rho_{0}\left(S_{0} \quad\left(\lambda y_{1}+\left(\begin{array}{ll}
1 & \lambda
\end{array}\right) y_{2}\right) \quad \pi\left(y_{0} \quad\left(\lambda y_{1}+\left(\begin{array}{ll}
1 & \lambda
\end{array}\right) y_{2}\right)\right)\right) \\
& \leqslant \lambda \rho_{0}\left(S_{0} \quad y_{1} \quad \pi\left(y_{0} \quad y_{1}\right)\right)+\left(\begin{array}{llll}
1 & \lambda
\end{array}\right) \rho_{0}\left(S_{0} \quad y_{2} \quad \pi\left(y_{0} \quad y_{2}\right)\right) .
\end{aligned}
$$

Since the multiobjective optimization problem (15) is convex, it may be solved by scalarization methods, i.e., in order to obtain Par eto solutions one can minimize a convex combination of $\rho_{0}$ and $\mathbb{E}$. Accordingly, take $w_{0}$ and $w_{1}$ non negative and such that $w_{0}+w_{1}=1$, let $\rho \quad w_{0} \rho_{0} \quad w_{1} \mathbb{E}$, and solve

\footnotetext{
${ }^{5}$ See Artzner et al. (1999), or verify that $\rho_{0}$ is decreasing from (3) and (9).
} 
$\left\{\begin{array}{l}\operatorname{Min} \rho\left(S_{0} \quad y \quad \pi\left(y_{0} \quad y\right)\right), \\ \pi\left(y_{0} \quad y\right) \leqslant S_{1} \\ 0 \leqslant y \leqslant y_{0} .\end{array}\right.$

Bearing in mind the ideas of the previous section, $\rho$ satisfies Assumption 1 and is coherent, since it is a convex combination of $\rho_{0}$ and $\mathbb{E}$.

It is worth remarking that the first (second) objective of (15) may be removed and the problem still fits in (17), because one can take $w_{0}=0$ and $w_{1}=1\left(w_{0}=1\right.$ and $\left.w_{1}=0\right)$.

Next we will give necessary and sufficient Karush Kuhn Tucker optimality conditions.

Theorem 2. Problem (17) is bounded and solvable. Moreover, the existence of $\left(\tau^{*}, z^{*}\right) \in \mathbb{R} \times L^{2}$ satisfying the following Karush Kuhn Tucker like conditions is necessary and sufficient to guarantee the optimality of $y^{*} \in L^{2}$.

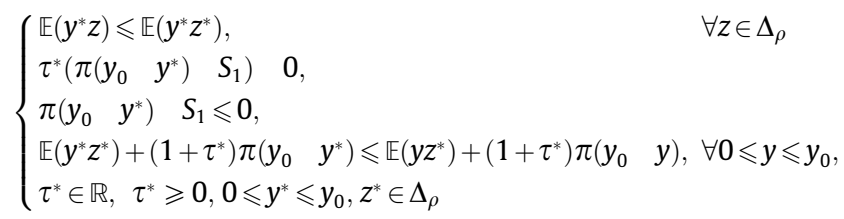

$\left(\tau^{*}, z^{*}\right)$ will be called KKT multiplier of (17).

Proof. The dimension of $L^{2}$ is finite due to the assumptions ( $\Omega$ is discrete and finite, see ( 1 ) and Footnote 3 ). Thus, the finite dimen sion of $L^{2}$ and the convexity of $\pi: L^{2} \rightarrow \mathbb{R}$ guarantees the continuity of $\pi$ (Luenberger, 1969). Similarly (6) and (7) show that $\rho$ is convex and therefore continuous. Besides, the last constraint of (17) shows that the feasible set is bounded, and therefore compact. Hence, the Weierstrass theorem shows that (17) is bounded and solvable. Finally, we will not prove the Karush Kuhn Tucker like conditions because an analogous proof may be found in Balbás et al. (2009).

A first important consequence is that one can give conditions ensuring that the solution of (17) remains the same if $\rho$ is replaced by a lower one. ${ }^{6}$ Hence we can give the first result guaranteeing the stability of the optimal insurance (retention) with respect to the risk measure.

Corollary 3. Suppose that $y^{*} \in L^{2}$ solves (17) and $\left(\tau^{*}, z^{*}\right)$ is a KKT multiplier. Take the coherent risk measure $\rho$ satisfying Assumption 1 and such that $\rho \leqslant \rho$. If $z^{*} \in \Delta_{\tilde{\rho}}$ and $\rho$ replaces $\rho$ then $y^{*} \in L^{2}$ still solves (17) and $\left(\tau^{*}, z^{*}\right)$ is still a KKT multiplier.

Proof. On the one hand, $y^{*}$ and $\left(\tau^{*}, z^{*}\right)$ satisfy (18). On the other hand, according to that properties given in the previous section, $\Delta_{\rho} \subset \Delta_{\rho}$ because $\rho \leqslant \rho$. Thus, $z^{*} \in \Delta_{\rho}$ implies that (18) still holds if $\Delta_{\rho}$ replaces $\Delta_{\rho}$.

Corollary 4. Suppose that $y_{\Gamma}^{*} \in L^{2}$ solves (17) and $\left(\tau_{\Gamma}^{*}, z_{\Gamma}^{*}\right)$ is a KKT multiplier for the risk measure $\Gamma$ of (12). Then $y_{\Gamma}^{*}$ still solves (17) and $\left(\tau_{\Gamma}^{*}, z_{\Gamma}^{*}\right)$ is still a KKT multiplier for every $\rho$ with $z_{\Gamma}^{*} \in \Delta_{\rho}$.

Proof. It trivially follows from the previous corollary and (13).

\footnotetext{
${ }^{6}$ With the notations of (15), notice that $\rho$ decreases if so does $\rho_{0}$, i.e.,

$\rho_{0} \geqslant \tilde{\rho}_{0} \Rightarrow w_{0} \rho_{0} \quad w_{1} \mathbb{E} \geqslant w_{0} \tilde{\rho}_{0} \quad w_{1} \mathbb{E}$.
}

Remark 1. With the notations of Corollary 4 , if $z_{\Gamma}^{*} \notin \Delta_{\rho}$ one still can look for a risk measure $\rho \geqslant \rho$ quite similar to $\rho$ and such that $z_{\Gamma}^{*} \in \Delta_{\rho}$, and therefore $y_{\Gamma}^{*}$ still solves (17) and $\left(\tau_{\Gamma}^{*}, z_{\Gamma}^{*}\right)$ is still a $K K T$ multiplier if one considers $\rho$. Indeed, it is sufficient to take the following convex and compact set, ${ }^{7}$

$\Delta_{\tilde{\rho}} \quad \operatorname{Co}\left(\Delta_{\rho} \cup\left\{z_{\Gamma}^{*}\right\}\right)$,

obviously associated with the risk measure

$\tilde{\rho}(y) \quad \operatorname{Max}\left\{\rho(y), \quad \mathbb{E}\left(y z_{\Gamma}^{*}\right)\right\}$

for every $y \in L^{2}$. For this reason hereafter the solution $y_{\Gamma}^{*} \in L^{2}$ of (17) for the risk measure $\Gamma$ of (12) will be called "stable optimal retention".

Remark 2. If the ceding company is also interested in maximizing the expected wealth and deals with problem (15), then $\Gamma$ may be replaced by $w_{0} \Gamma \quad w_{1} \mathbb{E}$ (with $w_{i} \geqslant 0, i=0,1$, and $w_{0}+w_{1}=1$ ). Indeed, in such a case, (11) and (14) show that

$\Delta_{w_{0} \Gamma w_{1} \mathbb{E}} \quad\left\{z \in L^{2} ; \mathbb{E}(z) \quad 1\right.$ and $\left.z \geqslant w_{1}\right\}$.

Obviously, Corollary 3 proves that if $y_{w_{0} \Gamma}^{*} \in L^{2}$ solves (15) and $\left(\tau_{w_{0} \Gamma}^{*}, z_{w_{0} \Gamma}^{*}\right)$ is a KKT multiplier for the risk measure $w_{0} \Gamma \quad w_{1} \mathbb{E}$ above, then $y_{w_{0} \Gamma}^{*}$ still solves (15) and $\left(\tau_{w_{0} \Gamma}^{*}, z_{w_{0} \Gamma}^{*}\right)$ is still a KKT mul tiplier for every $\rho$ such that $z_{w_{0} \Gamma}^{*} \in \Delta_{w_{0} \rho w_{1} \mathbb{E}}$. Furthermore, a new comment similar to Remark 1 applies.

\section{Characterizing and computing the stable optimal retention}

Let us give properties making it easier to verify the fulfillment of the inequalities of (18). To this purpose, and taking into account Corollary 4, Remark 2 and the first condition in (18), let us give an instrumental lemma.

Lemma 5. Suppose that $0 \leqslant y^{*} \leqslant y_{0}$ and $z^{*} \in \Delta_{w_{0} \Gamma-w_{1} \mathbb{E}}$ (see (20)). $\mathbb{E}\left(y^{*} z\right) \leqslant \mathbb{E}\left(y^{*} z^{*}\right)$ holds for every $z \in \Delta_{w_{0} \Gamma-w_{1} \mathbb{E}}$ if and only if

$y^{*}\left(\omega_{j}\right) \quad \operatorname{Max}\left\{y^{*}\left(\omega_{i}\right): i \quad 1,2, \ldots, n\right\}$,

holds for every $j=1,2, \ldots, n$ such that $z^{*}\left(\omega_{j}\right)>w_{1}$.

Proof. The inequality above holds if and only if $z^{*}$ solves the linear optimization problem

$$
\left\{\begin{array}{l}
\operatorname{Max} \sum_{i=1}^{n} y^{*}\left(\omega_{i}\right) z\left(\omega_{i}\right) p_{i}, \\
\sum_{i=1}^{n} z\left(\omega_{i}\right) p_{i} \quad 1, \\
w_{1} \leqslant z\left(\omega_{i}\right), \quad i \quad 1,2, \ldots, n .
\end{array}\right.
$$

According to the classical Karush Kuhn Tucker conditions, this is equivalent to the existence of $\mu_{0}, \mu_{1}, \ldots, \mu_{n} \in \mathbb{R}$ such that

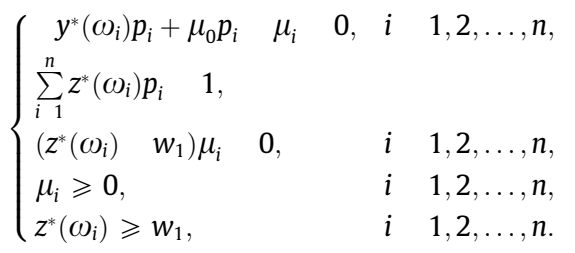

Hence, the result trivially follows if one takes

$\mu_{0} \operatorname{Max}\left\{y^{*}\left(\omega_{i}\right): i \quad 1,2, \ldots, n\right\}$

$\overline{{ }^{7} \text { As usual, }} \operatorname{Co}(A)$ denotes the convex hull of every set $A \subset L^{2}$. 
and

$\mu_{i} \quad\left(\mu_{0} \quad y^{*}\left(\omega_{i}\right)\right) p_{i}$,

$i=1,2, \ldots, n$.

Despite the fact that previous analyses are quite general, the solutions of (18) will depend on the specific assumptions one im poses. Henceforth we will assume that the reinsurer uses a linear premium principle. Actually, as indicated in the introduction, pre vious literature considering a general risk measure is scant, so it seems to be natural and of interest to analyze concrete problems by taking the most used premium principle, which is the expected value premium principle, i.e., there exists $k>1$ such that

$\pi(y) \quad k \mathbb{E}(y)$

for every $y \in L^{2}$. We will impose something strictly weaker, such as the existence of $z_{\pi} \in L^{2}$ such that

$\mathbb{P}\left(z_{\pi}>0\right) \quad 1$

$\mathbb{E}\left(z_{\pi}\right)>1$

and

$\pi(y) \quad \mathbb{E}\left(y z_{\pi}\right)$

for every $y \in L^{2}$.

Assumption 2. Henceforth we will assume the existence of $z_{\pi} \in L^{2}$ such that (22) (24) hold.

Nevertheless, it is worth pointing out that the previous develop ments are more general, and therefore they also apply to alterna tive premium principles.

From Assumption 2 the necessary and sufficient optimality con ditions (18) become

$$
\begin{cases}\mathbb{E}\left(y^{*} z\right) \leqslant \mathbb{E}\left(y^{*} z^{*}\right), & \forall z \in \Delta_{\rho}, \\ \tau^{*}\left(\mathbb{E}\left(\left(y_{0} \quad y^{*}\right) z_{\pi}\right) \quad S_{1}\right) \quad 0, & \\ \mathbb{E}\left(\left(y_{0} \quad y^{*}\right) z_{\pi}\right) \quad S_{1} \leqslant 0, & \\ \mathbb{E}\left(y^{*}\left(z^{*} \quad\left(1+\tau^{*}\right) z_{\pi}\right)\right) \leqslant \mathbb{E}\left(y\left(z^{*} \quad\left(1+\tau^{*}\right) z_{\pi}\right)\right), & \forall 0 \leqslant y \leqslant y_{0}, \\ \tau^{*} \in \mathbb{R}, \quad \tau^{*} \geqslant 0, \quad 0 \leqslant y^{*} \leqslant y_{0}, z^{*} \in \Delta_{\rho} . & \end{cases}
$$

Next let us present two simple lemmas. The first one simplifies the fourth condition of (25).

Lemma 6. Let $z^{*} \in L^{2}, y^{*} \in L^{2}$ with $0 \leqslant y^{*} \leqslant y_{0}$, and $\tau^{*} \in \mathbb{R}$. Then,

$\mathbb{E}\left(y^{*}\left(z^{*} \quad\left(1+\tau^{*}\right) z_{\pi}\right)\right) \leqslant \mathbb{E}\left(y\left(z^{*} \quad\left(1+\tau^{*}\right) z_{\pi}\right)\right)$,

holds for every $y \in L^{2}$ with $0 \leqslant y \leqslant y_{0}$ if and only if there exists $a$ partition

$\Omega \quad \Omega_{1} \cup \Omega_{2} \cup \Omega_{3}$,

such that

$\left\{\begin{array}{llll}z^{*}(\omega)>\left(1+\tau^{*}\right) z_{\pi}, & y^{*}(\omega) & 0, & \text { if } \omega \in \Omega_{1}, \\ z^{*}(\omega) \quad\left(1+\tau^{*}\right) z_{\pi}, & & & \text { if } \omega \in \Omega_{2}, \\ z^{*}(\omega)<\left(1+\tau^{*}\right) z_{\pi}, & y^{*}(\omega) & y_{0}(\omega), & \text { if } \omega \in \Omega_{3} .\end{array}\right.$

Proof. It is obvious if we realize that the solution of

$$
\left\{\begin{array}{l}
\operatorname{Min} \mathbb{E}\left(y\left(z^{*} \quad\left(1+\tau^{*}\right) z_{\pi}\right)\right) \\
0 \leqslant y \leqslant y_{0}
\end{array}\right.
$$

\footnotetext{
${ }^{8}$ Notice that (21) is a particular case of (24) that arises if $z_{\pi}$ remains constant and equals $k$.
}

must be as large as possible (i.e., must equal $y_{0}$ ) whenever $z^{*}\left(1+\tau^{*}\right) z_{\pi}<0$ and as small as possible (i.e., zero) if $z^{*}\left(1+\tau^{*}\right) z_{\pi}>0$, whereas its value is not relevant at all if $z^{*} \quad\left(1+\tau^{*}\right) z_{\pi}=0$.

Lemma 7. $y^{*}=0$ does not solve (17).

Proof. If $y^{*}=0$ solved (17) then (26) would lead to $z^{*} \geqslant\left(1+\tau^{*}\right) z_{\pi}$. Bearing in mind (4) and (23), and taking expectations, one has the contradiction $1 \geqslant\left(1+\tau^{*}\right) \mathbb{E}\left(z_{\pi}\right)>1$.

As already said the stop loss reinsurance is often obtained as the optimal retention (Balbás et al., 2009). Recall that $y \in L^{2}$ and ly ing between 0 and $y_{0}$ is said to be a stop loss reinsurance if there exists $\alpha \geqslant 0$ such that

$y \quad \begin{cases}y_{0}, & y_{0} \leqslant \alpha, \\ \alpha, & y_{0}>\alpha .\end{cases}$

Hereafter the random variable of (27) will be denoted by $y_{0}^{\alpha}$.

Corollary 4 and Remark 2 show the importance of solving (17) when $\rho \quad w_{0} \Gamma \quad w_{1} \mathbb{E}$, since the solution will generate a very stable optimal reinsurance contract.

Theorem 8. Consider problem (17) with the risk measure $w_{0} \Gamma \quad w_{1} \mathbb{E}$. Suppose that $\mathbb{P}\left(z_{\pi}>w_{1}\right) \quad 1 .{ }^{9}$

(a) There exists $\alpha^{*}>0$ such that $y_{0}^{\alpha^{*}}$ solves (17).

(b) Suppose that $y_{0}^{\alpha^{*}}$ solves (17), $\mathbb{P}\left(y_{0} \quad \alpha^{*}\right) \quad 0$ and $\left(\tau_{w_{0} \Gamma}^{*}, z_{w_{0} \Gamma}^{*}\right)$ is a KKT multiplier of (17). Then

$z_{w_{0} \Gamma}^{*} \begin{cases}w_{1}, & \text { if } y_{0}<\alpha^{*}, \\ \left(1+\tau_{w_{0} \Gamma}^{*}\right) z_{\pi}, & \text { if } y_{0}>\alpha^{*} .\end{cases}$

(c) Suppose that $y_{0}^{\alpha^{*}}$ solves (17), there is a unique $\omega_{i_{0}} \in \Omega$ with $y_{0}\left(\omega_{i_{0}}\right) \quad \alpha^{*}$ and $\left(\tau_{w_{0} \Gamma}^{*}, z_{w_{0} \Gamma}^{*}\right)$ is a KKT multiplier of (17). Then

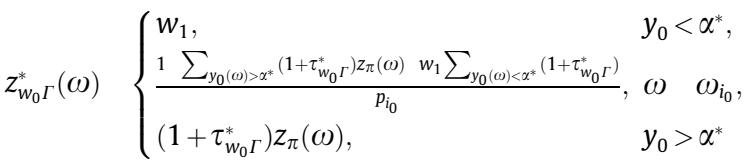

and

$$
\begin{aligned}
& \frac{1 \sum_{y_{0}(\omega)>\alpha^{*}}\left(1+\tau_{w_{0} \Gamma}^{*}\right) z_{\pi}\left(\omega_{i_{0}}\right) \quad w_{1} \sum_{y_{0}(\omega)<\alpha^{*}}\left(1+\tau_{w_{0} \Gamma}^{*}\right)}{p_{i_{0}}} \\
& \leqslant\left(1+\tau_{w_{0} \Gamma}^{*}\right) z_{\pi}\left(\omega_{i_{0}}\right),
\end{aligned}
$$

hold.

(d) Suppose that $y_{0}^{\alpha^{*}}$ solves (17) and $\left(\tau_{w_{0} \Gamma}^{*}, z_{w_{0} \Gamma}^{*}\right)$ is a KKT multi plier of (17). Suppose that $\rho$ is coherent and satisfies Assumption 1. If $z_{w_{0} \Gamma}^{*} \in \Delta_{w_{0} \rho} w_{1} \mathbb{E}$ then $y_{0}^{\alpha^{*}}$ solves (17) for $w_{0} \rho \quad w_{1} \mathbb{E}$.

\section{Proof}

(a) Take the solution $y^{*}$ of (17) whose existence is guaranteed by Theorem 2, and define

$$
\alpha^{*} \operatorname{Max}\left\{y^{*}\left(\omega_{i}\right): i \quad 1,2, \ldots, n\right\} .
$$

${ }^{9}$ (22) implies the fulfillment of this property whenever $w_{0}=1$. Since $w_{0} \leqslant 1$, the property holds if the reinsurer draws on the expected value premium principle, since then $z_{\pi}=k>1$. 
Lemma 7 implies that $\alpha^{*}>0$. Let us see that $y^{*} y_{0}^{\alpha^{*}}$. Indeed, $y^{*}$ being (17) feasible we have that $y^{*} \leqslant y_{0}$, so $\alpha^{*} \leqslant y_{0}(\omega)$ whenever $y^{*}(\omega)=\alpha^{*}$. Besides, if $y^{*}(\omega)<\alpha^{*}$ and $\left(\tau_{w_{0} \Gamma}^{*}, z_{w_{0} \Gamma}^{*}\right)$ is a KKT multiplier (its existence follows from Theorem 2), then the first condition in (25) and Lemma 5 lead to $z_{w_{0} \Gamma}^{*}(\omega) \quad w_{1}$. Hence, the fourth condition in (25), Expression (26) and $z_{\pi}>w_{1}$ lead to $y^{*}(\omega)=y_{0}(\omega)$, and therefore $y^{*} y_{0}^{\alpha^{*}}$.

(b) If $y_{0}^{\alpha^{*}}(\omega)<\alpha^{*}$ (or $y^{*}(\omega)<\alpha^{*}$ ) then the equality $z_{w_{0} \Gamma}^{*}(\omega) \quad w_{1}$ may be proved with the same arguments. Suppose that $y_{0}^{\alpha^{*}}(\omega) \quad \alpha^{*}$. Then, consider the partition of Lemma 6 and obviously $\omega \in \Omega_{2}$ or $\omega \in \Omega_{3}$, since $y_{0}^{\alpha^{*}}(\omega) \neq 0$. But $\omega \in \Omega_{3}$ would imply $y_{0}(\omega)=\alpha^{*}$, which cannot hold.

(c) As in the proof of (b), $z_{w_{0} \Gamma}^{*}(\omega) \quad w_{1}$ whenever $y_{0}^{\alpha^{*}}(\omega)<\alpha^{*}$. Suppose that $y_{0}^{\alpha^{*}}(\omega) \quad \alpha^{*}$. Then, consider the partition of Lemma 6 and obviously $\omega \in \Omega_{2}$ or $\omega \in \Omega_{3}$, since $y_{0}^{\alpha^{*}}(\omega) \neq 0$. But $\omega \in \Omega_{3}$ implies that $y_{0}(\omega)=\alpha^{*}$, and therefore $\omega \omega_{i_{0}}$. Thus, taking into account (4), we have (29). Finally, (30) comes from (26), because $\mathbb{P}\left(y_{0}^{\alpha^{*}} \quad 0\right) \quad 0$ implies that $z_{w_{0} \Gamma}^{*} \leqslant\left(1+\tau_{w_{0} \Gamma}^{*}\right) z_{\pi}$

(d) It trivially follows from Corollary 4 and Remark 2.

Remark 3. According to the previous theorem the "stable optimal retention" of Remark 1 is a stop loss reinsurance $y_{0}^{\alpha^{*}}$. Theorem 8 also provides the multiplier $z_{w_{0} \Gamma}^{*}$ (see (28) or (29)), so the condition $z_{w_{0} \Gamma}^{*} \in \Delta_{w_{0} \rho} w_{1} \mathbb{E}$ is very easy to verify in practical examples. Actu ally, we will see in the next section that the assumptions of state ments $8 \mathrm{~b}$ and $8 \mathrm{c}$ are usually fulfilled in practice.

Remark 4. Rockafellar et al. (2006) introduced the risk measure $C V a R_{\mu_{0}}, \mu_{0} \in(0,1)$ being the level of confidence. $C V a R_{\mu_{0}}$ is becom ing very important and popular among practitioners and research ers for its interesting properties. Indeed, it is coherent and expectation bounded (Rockafellar et al., 2006), and compatible with the second order stochastic dominance and the classical util ity functions (Ogryczak and Ruszczynski, 2002). ${ }^{10}$ Rockafellar et al. (2006) proved that

$\Delta_{\mathrm{CVaR}_{\mu_{0}}} \quad\left\{z \in L^{2} ; 0 \leqslant z \leqslant \frac{1}{1 \quad \mu_{0}}, \mathbb{E}(z) \quad 1\right\}$.

Consider $w_{0}=1$ (the expected wealth is not optimized by the ceding company). Thus, if $\rho \quad \mathrm{CVaR}_{\mu_{0}}$ in problem (17), then $y_{0}^{\alpha^{*}}$ will solve the problem (i.e., (31) will contain the random variable $z_{\Gamma}^{*}$ ) as long as

$\frac{1}{1 \quad \mu_{0}} \geqslant z_{\Gamma}^{*}$

which clearly holds for $\mu_{0}$ close enough to $100 \%$. Analogously, if the insurance company deals with problem (15) and $\rho_{0} \quad C V a R_{\mu_{0}}$, then the solution $y_{0}^{\alpha^{*}}$ of (15) for $w_{0} \Gamma \quad w_{1} \mathbb{E}$ will be still the solution for the $w_{0} C V a R_{\mu_{0}} \quad w_{1} \mathbb{E}$ as long as

$\frac{w_{0}}{1 \frac{\mu_{0}}{\mu_{0}}}+w_{1} \geqslant z_{w_{0} \Gamma}^{*}$,

which is also obvious for $w_{0}>0$ and $\mu_{0}$ large enough. An illustrative numerical example will be given in Section 5 .

\section{Algorithm and numerical experiment}

Next let us point out that the conditions of Theorem 8 usually hold in practice, and the stable optimal retention $y_{0}^{\alpha^{*}}$ and the

\footnotetext{
${ }^{10}$ Recall that the standard deviation is not compatible with the second orde stochastic dominance if asymmetries are involved (Ogryczak and Ruszczynski, 1999), and the stop-loss reinsurance obviously generates asymmetric results.
}

KKT multiplier $\left(\tau_{\Gamma}^{*}, z_{\Gamma}^{*}\right)$ may be easily calculated by drawing on an appropriate algorithm. The algorithm just tests the fulfillment of Theorem 8.

First of all we will introduce the algorithm and then we will present a numerical example. In order to simplify the exposition, in this section we will assume that $w_{1}=0$ (the expected wealth is not maximized, and only the risk level is minimized), though the extension for $w_{1}>0$ is straightforward.

Notice that, according to Theorem $8, y_{0}^{\alpha^{*}}$ and $\left(\tau_{\Gamma}^{*}, z_{\Gamma}^{*}\right)$ will be known once we compute $\alpha^{*}$ and $\tau_{\Gamma}^{*}$, i.e., we only have to estimate two real numbers.

In order to introduce the algorithm we will assume that

$\Omega \quad\left\{\omega_{1}, \omega_{2}, \ldots, \omega_{n}\right\} \subset \mathbb{R}$,

$0<\omega_{1}<\omega_{2}<\cdots<\omega_{n}$

and $y_{0}$ is the identity map, so

$\mathbb{P}\left(y_{0} \quad \omega_{i}\right) \quad p_{i}$,

$i=1,2, \ldots, n$. Actually, this is a particular framework strictly more restricted than that in Theorem 8 , but this is a standard simplifica tion in the literature about the optimal reinsurance problem. See, for instance, Gajec and Zagrodny, 2004; Kaluszka, 2005; Cai et al., 2008 , and many others, where the authors do not deal with the ori ginal probability space $(\Omega, \mathscr{F}, \mathbb{P})$, but with its image $\left(\mathbb{R}, \mathscr{B}, \mathbb{P}^{*}\right)$ by $y_{0}$, composed of the real line $\mathbb{R}$, the Borel $\sigma$ algebra $\mathscr{B}$ of $\mathbb{R}$, and the probability measure $\mathbb{P}^{*}$ given by

$\mathbb{P}^{*}(B) \quad \mathbb{P}\left(\omega \in \Omega ; y_{0}(\omega) \in B\right)$

for every Borel subset $B \in \mathscr{B}$. In such a particular case $y_{0}$ is replaced by the identity map. Besides, in practical situations insurers usually deal with $\left(\mathbb{R}, \mathscr{B}, \mathbb{P}^{*}\right)$ and the identity map too, which means that they do not distinguish different events leading to the same cost of claims. Finally, though the new setting (33) is much more re stricted than the original one, the simplification does not modify the computation of the solution of (17). Indeed, Theorem 8 guaran tees that we are looking for a stop loss reinsurance, and there obvi ously exists a one to one mapping between the stop loss contracts of the initial probability space $(\Omega, \mathscr{F}, \mathbb{P})$ and the stop loss contracts of its image $\left(\mathbb{R}, \mathscr{B}, \mathbb{P}^{*}\right)$.

Hence, assume (33) (35) and define

$\alpha_{\operatorname{Max}} \omega_{n}$

Obviously, $y_{0}^{\alpha_{\text {Max }}} \quad y_{0}$ is (17) feasible because $S_{1}>0$ and $\pi(0)=0$. Due to (22), the premium principle of (24) generates a strictly increasing function $\pi .{ }^{11}$ Consequently, $\pi\left(y_{0} \quad y_{0}^{\alpha}\right)$ strictly decreases as $\alpha$ grows. Consider a first case (Case_1) such that $\pi\left(y_{0}\right) \leqslant S_{1}$, which implies that $y_{0}^{\alpha}$ is (17) feasible for every $\alpha \geqslant 0$ and therefore we will consider $^{12}$

$\alpha_{\text {Min }} 0$.

If $\pi\left(y_{0}\right)>S_{1}$ then the continuity of $\alpha \rightarrow \pi\left(y_{0} \quad y_{0}^{\alpha}\right)$ implies the exis tence of a unique $\alpha_{\text {Min }} \in\left(0, \alpha_{\text {Max }}\right)$ such that

$\pi\left(\begin{array}{lll}y_{0} & y_{0}^{\alpha_{M i n}}\end{array}\right) \quad S_{1}$.

Let us distinguish two situations. Case_2 arises if $\alpha_{\operatorname{Min}} \notin \Omega$, in which case we will chose $i_{0}$ as the smallest subscript such that

$\pi\left(y_{0} \quad \omega_{i_{0}}\right)<S_{1}$.

Case_3 holds if $\alpha_{\operatorname{Min}} \quad \omega_{i_{0}} 1 \in \Omega$ for some $i_{0}$.

Obviously, for the three cases $y_{0}^{\alpha}$ is (17) feasible if and only if

$\alpha_{\text {Min }} \leqslant \alpha \leqslant \alpha_{\text {Max }}$

\footnotetext{
11 i.e., $\pi\left(y_{1}\right)<\pi\left(y_{2}\right)$ whenever $y_{1} \leqslant y_{2}$ and $y_{1} \neq y_{2}$.

12 Actually, Constraint $\pi\left(y_{0}-y\right) \leqslant S_{1}$ is redundant in this case, and may be removed in (17).
} 
Algorithm 1. Suppose that Case_1 holds. Lemma 6 implies that $y_{0}^{\alpha_{\text {Min }}}$ does not solve (17), so the stable optimal retention $y_{0}^{\alpha^{*}}$ satisfies $\pi\left(\begin{array}{ll}y_{0} & y_{0}^{\alpha^{*}}\end{array}\right)<S_{1}$ and the second condition in (18) leads to $\tau_{\Gamma}^{*} \quad 0$. Hence, we only have to estimate $\alpha^{*}$.

Step 1. Define

$$
\begin{gathered}
\alpha_{1} \frac{\omega_{1}}{2}, \alpha_{2} \quad \omega_{1}, \alpha_{3} \quad \frac{\omega_{1}+\omega_{2}}{2}, \alpha_{4} \quad \omega_{2}, \ldots, \alpha_{2 n} 1 \\
\frac{\omega_{n 1}+\omega_{n}}{2}, \alpha_{2 n} \quad \omega_{n} .
\end{gathered}
$$

Step 2. For $j=1$ to $n$ check whether $y_{0}^{\alpha_{2 j} 1}$ and

$$
z_{2 j 1}^{*}\left\{\begin{array}{l}
0, \quad \text { if } \omega<\alpha_{2 j 1}, \\
z_{\pi}, \quad \text { if } \omega \geqslant \alpha_{2 j 1},
\end{array}\right.
$$

satisfy (25) and (26). If these conditions are satisfied for some $y_{0}^{\alpha_{2 j}}$ then we will have the stable optimal retention and the KKT multi plier. The algorithm can stop since the stable optimal retention has been found.

Notice that two different values of $j$ cannot satisfy (25) and (26), since (22) implies that $\mathbb{E}\left(z_{2 j}^{*}\right)$ strictly decreases with $j$ and there fore (4) cannot hold two times. Furthermore, if these conditions hold for some $j$ then every $\alpha^{*} \in\left(\alpha_{2 j} 2, \alpha_{2 j}\right)$ will generate a stop loss stable optimal retention $y_{0}^{\alpha^{*}}$, since the same KKT multipliers $z_{2 j}^{*} 1$ and $\tau_{2 j 1}^{*} \quad 0$ will still apply.

Step 3. Suppose that Step 2 did not lead to the stable optimal retention. For $j=1$ to $n$ check whether $y_{0}^{\alpha_{2 j}}$ and

$z_{2 j}^{*}(\omega) \begin{cases}0, & \omega<\alpha_{2 j}, \\ \frac{1 \sum_{\omega>\alpha_{2 j}} z_{\pi}(\omega)}{p_{j}}, & \omega \quad \alpha_{2 j}, \\ z_{\pi}(\omega), & \omega>\alpha_{2 j},\end{cases}$

satisfy (25) and (26). Every time these conditions are satisfied we will have a solution of (17) for $\rho=\Gamma$. Notice that (26) will imply

$\frac{1 \sum_{\omega>\alpha_{2 j}} z_{\pi}\left(\alpha_{2 j}\right)}{p_{j}} \leqslant z_{\pi}\left(\alpha_{2 j}\right)$.

Algorithm 2. Suppose that Case_2 holds. Then proceed as in Algo rithm 1 with minor modifications in Steps 1 3. Now, in Step 1 we must define

$\alpha_{2 i_{0}} 1 \quad \frac{\alpha_{M i n}+\omega_{i_{0}}}{2}, \alpha_{2 i_{0}} \quad \omega_{i_{0}}, \alpha_{2 i_{0}+1} \quad \frac{\omega_{i_{0}}+\omega_{i_{0}+1}}{2}, \ldots$,

$\alpha_{2 n 1} \quad \frac{\omega_{n} 1+\omega_{n}}{2}, \alpha_{2 n} \quad \omega_{n}$.

Obviously, Steps 2 and 3 will start with $j=i_{0}$ rather than $j=1$.

Step 4. If Steps 2 and 3 did not lead to the stable optimal reten tion then we must address Step 4 so as to check the opti mality of $y_{0}^{\alpha_{M i n}}$. In this case $\tau_{\Gamma}^{*}>0$ may hold and we are in the conditions of Theorem $8 b$. We must verify whether $y_{0}^{\alpha_{\text {Min }}}, \tau_{\Gamma}^{*}$ and

$z_{\Gamma}^{*} \begin{cases}0, & \text { if } y_{0}<\alpha_{\text {Min }}, \\ \left(1+\tau_{\Gamma}^{*}\right) z_{\pi}, & \text { if } y_{0}>\alpha_{\text {Min }},\end{cases}$

satisfy (25) and (26) for some $\tau_{\Gamma}^{*} \geqslant 0$. Actually the only condition one must check is (4), i.e.,

$\left(1+\tau_{\Gamma}^{*}\right) \sum_{\omega_{i}>\alpha_{\text {Min }}} z_{\pi}\left(\omega_{i}\right) \quad 1$,

so the optimality of $y_{0}^{\alpha_{\text {Min }}}$ holds if and only if

$\tau_{\Gamma}^{*} \quad \frac{1}{\sum_{\omega_{i}>\alpha_{\text {Min }}} z_{\pi}\left(\omega_{i}\right)} \quad 1 \geqslant 0$.
Thus, Step 4 reduces to the verification of the inequality in (39). If this inequality holds then the equality in (39) provides us with $\tau_{\Gamma}^{*}$ (38) provides us with $z_{\Gamma}^{*}$, and $y_{0}^{\alpha_{\text {Min }}}$ is the stable optimal retention.

Algorithm 3. Suppose that Case_3 holds. Then proceed as in Algo rithm 2 with a minor modification in Step 1 . Now we must modify $\alpha_{2 i_{0} 1}$ according to

$$
\begin{aligned}
& \alpha_{2 i_{0} 1} \quad \frac{\omega_{i_{0}}+\omega_{i_{0}}}{2}, \alpha_{2 i_{0}} \quad \omega_{i_{0}}, \alpha_{2 i_{0}+1} \quad \frac{\omega_{i_{0}}+\omega_{i_{0}+1}}{2}, \ldots, \alpha_{2 n 1} \\
& \frac{\omega_{n 1}+\omega_{n}}{2}, \alpha_{2 n} \quad \omega_{n} \text {. }
\end{aligned}
$$

Once again, Step 2 and Step 3 will start with $j=i_{0}$.

Step 5. We still have to check the optimality of $y_{0}^{\alpha_{\text {Min }}} \quad y_{0}^{\omega_{i_{0}}}{ }^{1}$. This retention level is optimal if and only if we can find $\tau_{\Gamma}^{*} \geqslant 0$ such that $y_{0}^{\omega_{i_{0}}{ }^{1}}, \tau_{\Gamma}^{*}$ and

$z_{\Gamma}^{*}(\omega) \begin{cases}0, & \omega<\omega_{i_{0}} 1, \\ \frac{1 \sum_{\omega>\omega_{i_{0}} 1}\left(1+\tau_{\Gamma}^{*}\right) z_{\pi}(\omega)}{p_{i_{0}} 1}, & \omega \quad \omega_{i_{0}} 1, \\ \left(1+\tau_{\Gamma}^{*}\right) z_{\pi}(\omega), & \omega>\omega_{i_{0}} 1,\end{cases}$

satisfy (25) and (26). The existence of $\tau_{\Gamma}^{*}$ is easy to verify, because, bearing in mind the findings of Sections 3 and 4, one only needs to check the conditions

$$
\begin{aligned}
& 0 \leqslant 1 \sum_{\omega>\omega_{i_{0}} 1}\left(1+\tau_{\Gamma}^{*}\right) z_{\pi}(\omega), \\
& \frac{1 \sum_{\omega>\omega_{i_{0}} 1}\left(1+\tau_{\Gamma}^{*}\right) z_{\pi}\left(\omega_{i_{0}} 1\right)}{p_{i_{0}} 1} \leqslant\left(1+\tau_{\Gamma}^{*}\right) z_{\pi}\left(\begin{array}{ll}
\omega_{i_{0}} & 1
\end{array}\right)
\end{aligned}
$$

and

$$
1 \sum_{\omega>\omega_{i_{0}} 1}\left(1+\tau_{\Gamma}^{*}\right) z_{\pi}(\omega)+\left(1+\tau_{\Gamma}^{*}\right) \sum_{\omega>\omega_{i_{0}} 1} z_{\pi}(\omega) \mathbb{P}(\omega) \quad 1 .
$$

Equality (43) yields $\tau_{\Gamma}^{*}$, and then the inequalities (41) and (42) are equivalent to

$\tau_{\Gamma}^{*} \leqslant \frac{1}{\sum_{\omega>\omega_{i_{0}}} z_{\pi}(\omega)} \quad 1$

and

$$
\tau_{\Gamma}^{*} \geqslant \frac{1}{z_{\pi}\left(\omega_{i_{0}} 1\right)\left(p_{i_{0}} 1+1\right)} \quad 1,
$$

respectively. Thus, Step 4 reduces to the computation of $\tau_{\Gamma}^{*}$ by means of (43) and then the verification of the inequalities $\tau_{\Gamma}^{*} \geqslant 0$ (44) and (45). If the three inequalities hold then (40) provides us with the multiplier and $y_{0}^{\alpha_{\text {Min }}}$ is the stable optimal retention.

Remark 5. Notice that the existence of solution of (17) and the findings of Sections 3 and 4 show that at least one of the three algorithms must generate a stable optimal retention.

Remark 6. As said above, notice that the algorithm just tests the fulfillment of Theorem 8 , and consequently it is not very time con suming. Actually, it is a linear time algorithm, in the sense that there is a linear relationship between its computational complexity and the number of realizations of the global cost $y_{0}$.

Next let us present a simple numerical example. Our only objective is to illustrate how to use the algorithm in practical situations. 
Example 1. Suppose that $y_{0}$ can reach the values $100,200,300$, 400 and 500 with a similar probability 0.2 . Suppose that the reinsurer uses the expected value premium principle with a price $80 \%$ higher than the expected claims, i.e.

$\pi(y) \quad 1.8 \mathbb{E}(y)$.

Suppose finally that the ceding company does not impose any bud get constraint, i.e., we are in Case_1 above. With the notations of Algorithm 1, define

$$
\alpha_{1} \quad 50, \alpha_{2} \quad 100, \alpha_{3} \quad 150, \alpha_{4} \quad 200, \ldots, \alpha_{9} \quad 450, \alpha_{10} \quad 500 .
$$

In Step 2 we have to check the optimality of five stop loss con tracts. The first one is $y_{0}^{50}$. Consider

$z_{1}^{*} \quad \begin{cases}0, & \text { if } \omega<50, \\ 1.8, & \text { if } \omega \geqslant 50 .\end{cases}$

Obviously, $z_{1}^{*}$ remains constant and equals 1.8 , so it is not in the set $\Delta_{\Gamma}$ of (11). Then, $y_{0}^{50}$ is not a stable optimal retention. If one repeats the analysis with the four remaining "candidates" then similar re sults apply, so Step 2 does not generate any stable optimal retention.

In Step 3 we have to check the optimality of the remaining five stop loss contracts. The first one is $y_{0}^{100}$, and (36) gives

$$
z_{2}^{*}\left\{\begin{array}{cl}
0, & \text { if } \omega<100, \\
2.2, & \text { if } \omega 100, \\
1.8, & \text { if } \omega>100,
\end{array}\right.
$$

which do not belong to $\Delta_{\Gamma}$. Repeat the exercise with the remaining values of $\alpha$, and for $\alpha=200$ we get

$$
z_{4}^{*}\left\{\begin{array}{cl}
0, & \text { if } \omega<200, \\
0.4, & \text { if } \omega 200, \\
1.8, & \text { if } \omega>200,
\end{array}\right.
$$

which implies that $y_{0}^{200}$ is not a stable optimal retention either. Analogously, for $\alpha=300$ we get

$$
z_{6}^{*} \begin{cases}0, & \text { if } \omega<300, \\ 1.4, & \text { if } \omega 300, \\ 1.8, & \text { if } \omega>300\end{cases}
$$

and $y_{0}^{300}$ is the "stable optimal retention" we are looking for. It is easy to check that $y_{0}^{400}$ and $y_{0}^{500}$ are not stable optimal retentions. In fact, for $y_{0}^{400}$ one obtains

$$
z_{8}^{*} \begin{cases}0, & \text { if } \omega<400, \\ 3.2, & \text { if } \omega 400, \\ 1.8, & \text { if } \omega>400\end{cases}
$$

and this multiplier is not feasible because (37) does not hold. An analogous caveat arises for $y_{0}^{500}$.

Reinsurance $y_{0}^{300}$ will be the optimal retention for many risk measures. For instance, if one considers $\rho \quad \mathrm{CVaR}_{\mu_{0}}$, according to (32) $y_{0}^{300}$ solves the problem if

$$
\frac{1}{1 \quad \mu_{0}} \geqslant 1.8 \text {, }
$$

which holds for $\mu_{0} \geqslant 0.45$ (or $\mu_{0} \geqslant 45 \%$ ), and, in particular, for the usual values of this parameter in the industry, which are higher than $90 \%$. Finally, it is worthwhile to point out that the role of the $\mathrm{CVaR}_{\mu_{0}}$ may be also played by many other important risk measures in actuarial sciences, such as, WCVaR, DPT, Wang, etc.

\section{Conclusions}

The optimal reinsurance problem is a classic topic in actuarial theory. Since coherent and expectation bounded risk measures are becoming very important in Finance and Insurance, recent ap proaches deal with them so as to address the optimal reinsurance problem. However, there is no consensus about the risk measure that one must use, since every risk measure presents advantages and shortcomings when compared with others.

This article analyzes the "stability in the large" of the optimal reinsurance with respect to the risk measure that the insurer uses. It has been pointed out that there is a "stable optimal retention" that will show no sensitivity, insofar as it will solve the optimal reinsurance problem for many risk measures, providing a very ro bust reinsurance plan. For the expected value premium principle this stable optimal retention is a stop loss contract, and it is easy to compute in practice. A fast linear time algorithm has been given and a numerical example presented. The approach is general en ough. Actually, if desired, the analysis permits us to incorporate both budget constraints and the simultaneous maximization of the ceding company expected wealth.

\section{Acknowledgments}

Research partially supported by "RD_Sistemas $S A$ ", "Comunidad Autónoma de Madrid" (Spain), Grant S2009/ESP 1594, and "MEyC" (Spain), Grant ECO2009 14,457 C04.

The authors sincerely thank the reviewers for their useful comments.

The usual caveat applies.

\section{References}

Annaert, J., Van Osselaer, S., Verstraete, B., 2009. Performance evaluation of portfolio insurance strategies using stochastic dominance criteria. Journal of Banking and Finance 33, 272-280.

Arrow, K.J., 1963. Uncertainty and the welfare of medical care. American Economic Review 53, 941-973.

Artzner, P., Delbaen, F., Eber, J.M., Heath, D., 1999. Coherent measures of risk Mathematical Finance 9, 203-228.

Balbás, A., Balbás, B., Heras, A., 2009. Optimal reinsurance with general risk measures. Insurance: Mathematics and Economics 44, 374-384.

Balbás, A., Balbás, R., Garrido, J., 2010. Extending pricing rules with general risk functions. European Journal of Operational Research 201, 23-33.

Benati, S., 2003. The optimal portfolio problem with coherent risk measure constraints. European Journal of Operational Research 150, 572-584.

Bernard, C., Tian, W., 2009. Optimal reinsurance arrangements under tail risk measures. Journal of Risk and Insurance 76 (3), 709-725.

Borch, K., 1960. An attempt to determine the optimum amount of stop loss reinsurance. Transactions of the 16th International Congress of Actuaries I, 597610.

Burgert, C., Rüschendorf, L., 2006. Consistent risk measures for portfolio vectors. Insurance: Mathematics and Economics 38 (2), 289-297.

Cai, J., Tan, K.S., 2007. Optimal retention for a stop loss reinsurance under the VaR and CTE risk measures. ASTIN Bulletin 37 (1), 93-112.

Cai, J., Tan, K.S., Weng, C., Zhang, Y., 2008. Optimal reinsurance under VaR and CTE risk measures. Insurance: Mathematics and Economics 43, 185-196.

Centeno, M.L., Simoes, O., 2009. Optimal reinsurance. Revista de la Real Academia de Ciencias, RACSAM 103 (2), 387-405.

Cherny, A.S., 2006. Weighted V@R and its properties. Finance and Stochastics 10 367-393.

Deprez, O., Gerber, U., 1985. On convex principles of premium calculation. Insurance: Mathematics and Economics 4, 179-189.

Frittelli, M., Scandolo, G., 2005. Risk measures and capital requirements for processes. Mathematical Finance 16 (4), 589-612.

Gajec, L., Zagrodny, D., 2004. Optimal reinsurance under general risk measures. Insurance: Mathematics and Economics 34, 227-240.

Goovaerts, M., Kaas, R., Dhaene, J., Tang, Q., 2004. A new classes of consistent risk measures. Insurance: Mathematics and Economics 34, 505-516.

Kaluszka, M., 2005. Optimal reinsurance under convex principles of premium calculation. Insurance: Mathematics and Economics 36, 375-398.

Konno, H., Akishino, K., Yamamoto, R., 2005. Optimization of a long-short portfolio under non-convex transaction costs. Computational Optimization and Applications 32, 115-132.

Luenberger, D.G., 1969. Optimization by Vector Spaces Methods. John Wiley \& Sons.

Mansini, R., Ogryczak, W., Speranza, M.G., 2007. Conditional value at risk and related linear programming models for portfolio optimization. Annals of Operations Research 152, 227-256.

Miller, N., Ruszczynski, A., 2008. Risk-adjusted probability measures in portfolio optimization with coherent measures of risk. European Journal of Operational Research 191, 193-206. 
Nakano, Y., 2004. Efficient hedging with coherent risk measure. Journal of Mathematical Analysis and Applications 293, 345-354.

Ogryczak, W., Ruszczynski, A., 1999. From stochastic dominance to mean risk models: Semideviations and risk measures. European Journal of Operational Research 116, 33-50.

Ogryczak, W., Ruszczynski, A., 2002. Dual stochastic dominance and related mean risk models. SIAM Journal on Optimization 13, 60-78.

Rockafellar, R.T., Uryasev, S., Zabarankin, M., 2006. Generalized deviations in risk analysis. Finance and Stochastics 10, 51-74.
Samuelson, P.A., 1947. The Foundations of Economic Analysis. Harvard University Press.

Schied, A., 2007. Optimal investments for risk- and ambiguity-averse preferences: A duality approach. Finance and Stochastics 11, 107-129.

Wang, S.S., 2000. A class of distortion operators for pricing financial and insurance risks. Journal of Risk and Insurance 67, 15-36. 